
\title{
25 Research Soure \\ The decrease of CD4 T cell is associated with mortality in critical inpatients with COVID-19
}

\author{
Jun Xiong \\ Wuhan University Renmin Hospital \\ Mei Yang \\ Wuhan University Renmin Hospital \\ Weize Yang \\ Wuhan University Renmin Hospital \\ Xiaomin Luo \\ Wuhan University Renmin Hospital \\ $\mathrm{Ke} \mathrm{Hu}$ \\ Wuhan University Renmin Hospital \\ qingyan zhao ( $\nabla$ ruyan71@163.com ) \\ Wuhan University Renmin Hospital
}

\section{Research}

Keywords: COVID-19, risk factors, cellular immunity, mortality

Posted Date: May 27th, 2020

DOl: https://doi.org/10.21203/rs.3.rs-30057/v1

License: (c) (i) This work is licensed under a Creative Commons Attribution 4.0 International License.

Read Full License 


\section{Abstract}

Background The 2019 novel coronavirus (SARS-CoV-2) has caused an outbreak in the world. The critically ill patients had a high mortality. However, the possible risk factors of critical patients with coronavirus disease 2019 (COVID-19) are not fully known. We aim to investigate the risk factors in critical patients with COVID-19 and to address their role in predicting disease progression.

Methods In this single-centered, retrospective, observational study, we enrolled 91 critically ill adult patients with COVID-19 in Renmin Hospital of Wuhan University between Jan 20, 2020 and Feb 28, 2020. Data were collected using a standard method including clinical records and laboratory findings.

Results 39 patients $(42.9 \%)$ were dead and 52 patients $(57.1 \%)$ were cured and discharged before Mar 22, 2020. CD4 T cell count, CD8 T cell count and glomerular filtration rate were significantly lower in nonsurvivors than in survivors. However, the non-survivors presented a higher proportion of D-dimer, Cardiac troponin and immunoglobulin $\mathrm{G}$ than in survivors. Intravenous immunoglobulin was more common in survivors than in non-survivors. On multivariate analysis, D-dimer $(\mathbb{1} \mu \mathrm{g} / \mathrm{mL}, \mathrm{OR}=9.53,95 \% \mathrm{Cl}, 2.53-$

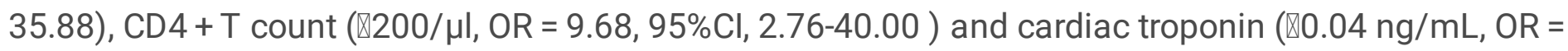
$5.73,95 \% \mathrm{Cl}, 1.86-17.66)$ were independent risk factors for mortality.

Conclusion The decrease of CD4 T cell is associated with higher risk mortality in critical inpatients with COVID-19. Intravenous immunoglobulin was more common in survivors than in non-survivors.

\section{Introduction}

At present, the novel severe acute respiratory syndrome coronavirus 2 (SARS-CoV-2) has outbreak and spread to many countries in the world. The current best estimate is that about $81 \%$ of people with coronavirus disease 2019 (COVID-19) have mild disease and never require hospitalization [1]. In s singlecenter case series of 138 hospitalized patients with COVID-19 in Wuhan, results showed that the mortality of patients was 4.3\% [2]. However, in another study, Yang reported that the mortality was $62 \%$ among critically ill patients with COVID-19 and $81 \%$ among those requiring mechanical ventilation in Wuhan [3]. Zhou and colleagues provide further insight into the mortality risk of critical inpatients with COVID-19. They found that older age, high sequential organ failure assessment (SOFA) score, and d-dimer greater than $1 \mu \mathrm{g} / \mathrm{L}$ are associated with the mortality risk of critical inpatients. In this study, not all laboratory tests were done, including lactate dehydrogenase, inflammatory and immune factors. Studies have suggested that a substantial decrease in the levels of lymphocytes indicates that coronavirus consumes many immune cells and inhibits the body's cellular immune function [4, 5]. Damage to T lymphocytes might be an important factor leading to exacerbations of patients [6]. In this study, we aim to investigate the risk factors in critical patients with COVID-19 and to address their role in predicting disease progression.

\section{Methods}


This single-centre, retrospective, observational study was done at Renmin Hospital of Wuhan University (Wuhan, China). 91 critical inpatients with COVID-19 from January 20, 2020 to Feb 28, 2020 were enrolled. This study was approved by the Ethics Committee of Renmin Hospital of Wuhan University. All critical inpatients were diagnosed with SARS-CoV-2 pneumonia, according to WHO interim guidance [7]. All cases matched clinical classification of critical type.

\section{Data collection}

The demographic characteristics, clinical records, initial hospitalized laboratory findings, computed tomography and outcome data were extracted from electronic medical records using the standardised data collection form. Two experienced clinicians entered data and reviewed the data. We collected data on age, sex, chronic medical histories (hypertension, chronic cardiac disease, chronic pulmonary disease, diabetes), the first symptoms, electrocardiogram and vital signs (heart rate, respiratory rate and Oxygen saturation), laboratory values on admission (blood routine examination, coagulation function, biochemical criterion, humoral immunity, cellular immunity, myocardial injury index and other indexes), treatment (antiviral drug: Arbidol/ Ribavirin/ interferon a), intravenous immunoglobulin, Chinese patent drug (XueBiJing/ TanReqing/ YanHuNing), Methylprednisolone), as well as living status.

\section{Outcomes}

The outcome was death or discharged. Discharge standard were defined according to the guidance of New coronavirus pneumonia prevention and control program in China (6th edition) [8]. Critical patient selection criteria (at least one of the following criteria): Pulse oxygen saturation $\leq 93 \%$ in resting state or shortness of breath (respiratory rate $\geq 30 \mathrm{bpm}$, or CT scan of the lungs suggesting that a lesion progression greater than $50 \%$ within 48 hours.

\section{Statistical analysis}

Continuous variables are expressed as means \pm standard deviation. The statistical differences were made using the unpaired and two-tailed t-test. Categorical data were compared using Pearson Chi-Square test or a Fisher exact test when expected values were less than 5 cases. Binary logistic regression analyses were used to assess the possible death predictors. Prespecified relevant clinical data was tested first in a univariate and then in a multivariate regression model by LR with a forward stepwise variable selection. $P$ value $<0.05$ was considered to indicate a statistically significant difference. The statistical analysis was performed using the SPSS 22.0 software.

\section{Results}

Baseline and treatment characteristics 
A total of 91 critically ill patients with critical COVID-19 were included in this study. 39 patients (42.9\%) were dead and 52 patients (57.1\%) were cured and discharged before Mar 22, 2020. The mean age was $63.9 \pm 14.1$ years (range, $29-85$ years), and $52(57.1 \%)$ were male. Baseline characteristics of the survivors and death are shown in Table 1. Overall, there was no significant difference in the proportion of hypertension, diabetes, chronic obstructive pulmonary disease (COPD), first symptoms and respiratory rate between survival group and death group. In addition to common symptoms such as fever, $11 \%$ of patients had diarrhea and $4.4 \%$ had nausea and vomiting, and one patient in death group has diarrhea as single symptom. The arrhythmic events, such as sinus tachycardia and atrial or ventricular arrhythmia showed no difference between the two groups. Furthermore, there was no significant difference abnormal ST segment and T wave between the two groups. There was no significant difference in the treatment of antiviral drug, Chinese patent drug (XueBiJing/ TanReqing/ YanHuNing) and Methylprednisolone between survival group and death group. However, intravenous immunoglobulin was more common in survivors than in death $(p=0.009)$. 
Table 1

Baseline and treatment characteristics

$\begin{array}{llll}\text { All patient } & \begin{array}{l}\text { Survival group }(n= \\ \text { 52) }\end{array} & \begin{array}{l}\text { Death } \\ \text { group }\end{array} & \begin{array}{l}P \\ \text { value } \\ (n=91)\end{array} \\ & & (n=39) & \end{array}$

Clinical characteristics and comorbidities

\begin{tabular}{|c|c|c|c|c|}
\hline Age(years) & $\begin{array}{l}63.91 \pm \\
14.1\end{array}$ & $59.1 \pm 13.2$ & $\begin{array}{l}70.4 \pm \\
12.9\end{array}$ & $\triangle 0.001$ \\
\hline$\otimes 50$ & $\begin{array}{l}16 \\
(17.6 \%)\end{array}$ & $14(26.9 \%)$ & $2(5.1 \%)$ & 0.011 \\
\hline $50-69$ & $\begin{array}{l}40 \\
(44.0 \%)\end{array}$ & 28 (53.8\%) & $\begin{array}{l}12 \\
(30.8 \%)\end{array}$ & 0.028 \\
\hline$\geq 70$ & $\begin{array}{l}35 \\
(38.5 \%)\end{array}$ & 10 (19.2\%) & $\begin{array}{l}25 \\
(64.1 \%)\end{array}$ & $\varangle 0.001$ \\
\hline Male & $\begin{array}{l}52 \\
(57.1 \%)\end{array}$ & 27 (51.9\%) & $\begin{array}{l}25 \\
(64.1 \%)\end{array}$ & 0.245 \\
\hline
\end{tabular}

Comorbidities

Number of comorbidities

\begin{tabular}{|c|c|c|c|c|}
\hline 0 & $\begin{array}{l}42 \\
(46.2 \%)\end{array}$ & $29(55.8 \%)$ & $\begin{array}{l}13 \\
(33.3 \%)\end{array}$ & 0.034 \\
\hline 1 & $\begin{array}{l}25 \\
(27.5 \%)\end{array}$ & $13(25.0 \%)$ & $\begin{array}{l}12 \\
(30.8 \%)\end{array}$ & 0.542 \\
\hline$\otimes 1$ & $\begin{array}{l}24 \\
(26.4 \%)\end{array}$ & $10(19.2 \%)$ & $\begin{array}{l}14 \\
(35.9 \%)\end{array}$ & 0.074 \\
\hline Hypertension & $\begin{array}{l}33 \\
(36.2 \%)\end{array}$ & $15(28.8 \%)$ & $\begin{array}{l}18 \\
(46.2 \%)\end{array}$ & 0.089 \\
\hline Coronary heart disease & $\begin{array}{l}12 \\
(13.2 \%)\end{array}$ & $3(5.8 \%)$ & $9(23.1 \%)$ & 0.026 \\
\hline Chronic obstructive pulmonary disease & $6(6.6 \%)$ & $2(3.8 \%)$ & $4(10.3 \%)$ & 0.396 \\
\hline Diabetes & $9(9.9 \%)$ & $6(11.5 \%)$ & $3(7.7 \%)$ & 0.727 \\
\hline \multicolumn{5}{|l|}{ Symptoms } \\
\hline Fever & $\begin{array}{l}74 \\
(81.3 \%)\end{array}$ & $42(80.8 \%)$ & $\begin{array}{l}32 \\
(82.1 \%)\end{array}$ & 0.877 \\
\hline Cough & $\begin{array}{l}42 \\
(46.2 \%)\end{array}$ & $23(44.2 \%)$ & $\begin{array}{l}19 \\
(48.7 \%)\end{array}$ & 0.671 \\
\hline
\end{tabular}




\begin{tabular}{|c|c|c|c|c|}
\hline & $\begin{array}{l}\text { All patient } \\
(n=91)\end{array}$ & $\begin{array}{l}\text { Survival group }(\mathrm{n}= \\
52 \text { ) }\end{array}$ & $\begin{array}{l}\text { Death } \\
\text { group } \\
(n=39)\end{array}$ & $\begin{array}{l}P \\
\text { value }\end{array}$ \\
\hline Sputum & $\begin{array}{l}14 \\
(15.4 \%)\end{array}$ & $5(9.6 \%)$ & $9(23.1 \%)$ & 0.069 \\
\hline Chest congestion & $\begin{array}{l}33 \\
(36.3 \%)\end{array}$ & $20(38.5 \%)$ & $\begin{array}{l}13 \\
(33.3 \%)\end{array}$ & 0.615 \\
\hline Dyspnea & $\begin{array}{l}18 \\
(19.8 \%)\end{array}$ & $8(15.4 \%)$ & $\begin{array}{l}10 \\
(25.6 \%)\end{array}$ & 0.224 \\
\hline Fatigue & $\begin{array}{l}33 \\
(36.3 \%)\end{array}$ & $15(28.8 \%)$ & $\begin{array}{l}18 \\
(46.2 \%)\end{array}$ & 0.089 \\
\hline Diarrhea & $\begin{array}{l}10 \\
(11.0 \%)\end{array}$ & $5(9.6 \%)$ & $5(12.8 \%)$ & 0.629 \\
\hline Nausea or vomiting & $4(4.4 \%)$ & $3(5.8 \%)$ & $1(2.6 \%)$ & 0.632 \\
\hline \multicolumn{5}{|l|}{ Vital signs } \\
\hline Respiratory rate( $\geq 30 \mathrm{bpm})$ & $\begin{array}{l}29 \\
(31.9 \%)\end{array}$ & $14(26.9 \%)$ & $\begin{array}{l}15 \\
(38.5 \%)\end{array}$ & 0.242 \\
\hline Oxygen saturation( $\leq 93 \%)$ & $70(77 \%)$ & $36(69.2 \%)$ & $\begin{array}{l}34 \\
(87.2 \%)\end{array}$ & 0.044 \\
\hline \multicolumn{5}{|l|}{ Electrocardiogram } \\
\hline Sinus tachycardia & $\begin{array}{l}15 \\
(16.5 \%)\end{array}$ & $6(11.5 \%)$ & $9(23.1 \%)$ & 0.227 \\
\hline Atrial or ventricular arrhythmia & $\begin{array}{l}10 \\
(11.0 \%)\end{array}$ & $6(11.5 \%)$ & $4(10.3 \%)$ & 0.691 \\
\hline Abnormal ST segment and T wave & $\begin{array}{l}19 \\
(20.9 \%)\end{array}$ & $12(23.1 \%)$ & $7(17.9 \%)$ & 0.369 \\
\hline \multicolumn{5}{|l|}{ Treatment } \\
\hline $\begin{array}{l}\text { Antiviral drug (Arbidol/ Ribavirin/ } \\
\text { interferon a) }\end{array}$ & $\begin{array}{l}85 \\
(93.4 \%)\end{array}$ & $49(94.2 \%)$ & $\begin{array}{l}36 \\
(92.3 \%)\end{array}$ & 0.715 \\
\hline Intravenous immunoglobulin & $\begin{array}{l}56 \\
(61.5 \%)\end{array}$ & $38(73.1 \%)$ & $\begin{array}{l}18 \\
(64.1 \%)\end{array}$ & 0.009 \\
\hline $\begin{array}{l}\text { Chinese patent drug } \\
\text { (XueBiJing/ TanReqing/ YanHuNing) }\end{array}$ & $\begin{array}{l}77 \\
(84.6 \%)\end{array}$ & $42(80.8 \%)$ & $\begin{array}{l}35 \\
(89.7 \%)\end{array}$ & 0.240 \\
\hline Methylprednisolone & $\begin{array}{l}77 \\
(84.6 \%)\end{array}$ & $41(78.8 \%)$ & $\begin{array}{l}36 \\
(92.3 \%)\end{array}$ & 0.140 \\
\hline
\end{tabular}


Compared with the survival group, patients in death group were older $(70.4 \pm 12.9$ years vs $59.1 \pm 13.2$ years, $p<0.001)$, and more likely to have low Oxygen saturation ( $\leq 93 \%, 87.2 \%$ vs $69.2 \%), p=0.044$ ). Moreover, coronary heart disease ( $23.1 \%$ vs $5.8 \%$ ) was present more often in non-survival group ( $p=$ 0.026).

\section{Laboratory findings}

The laboratory findings are shown in Table 2. Baseline lymphocyte count $(\mathbb{1 . 1} 1 \times 109 / \mathrm{L}, 84.6 \% \mathrm{vs} 63.5 \%$,

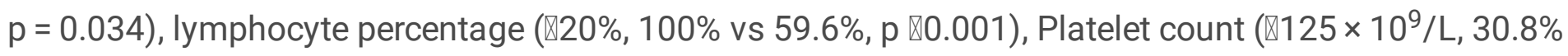
vs $11.5 \%, p=0.026)$ were significantly lower and white blood cell count $(\mathbb{2} .5 \times 109 / \mathrm{L}, 46.2 \%$ vs $13.5 \%, p=$ $0.001)$ and neutrophile granulocyte percentage $(\varangle 75 \%, 67.0 \%$ vs $46.2 \%, p \otimes 0.001)$ were significantly higher

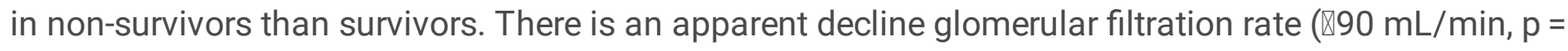
$0.005 ; \varangle 60 \mathrm{~mL} / \mathrm{min}, \mathrm{p}=0.033$ ) in the death group and only 2 of 91 patients had a previous history of renal dysfunction. Furthermore, cellular immunity, such as CD3 T cell count $(\varangle 723 / \mu \mathrm{l}, 89.7 \%$ vs $59.6 \%, p=$ $0.002)$, CD 4 T cell count $(\varangle 404 / \mu \mathrm{l}, 89.7 \%$ vs $51.9 \%$, $\mathrm{p} \otimes 0.001 ; \varangle 200 / \mu \mathrm{l}, 64.1 \%$ vs $23.1 \%$, $\mathrm{p} \otimes 0.001)$, and CD8 T

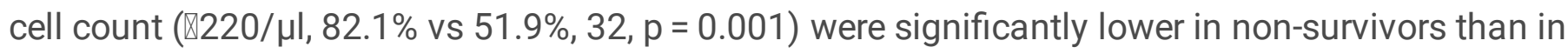
survivors. 
Table 2

Laboratory findings

$\begin{array}{llll}\text { All patient } & \begin{array}{l}\text { Survival } \\ \text { group }\end{array} & \begin{array}{l}\text { Death } \\ \text { group }\end{array} & \begin{array}{l}P \\ \text { value }\end{array} \\ (n=91) & (n=52) & (n=39) & \end{array}$

Blood routine examination

White blood cell count $\left(\times 10^{9} / \mathrm{L}\right)$

$\nabla 9.5$

25

$(27.5 \%)$

$7(13.5 \%)$

$18(46.2 \%) \quad 0.001$

$\otimes 3.5$

16

$(17.6 \%)$

$12(23.1 \%)$

$4(10.3 \%) \quad 0.164$

Neutrophile granulocyte $(₫ 75 \%)$

61

$(67.0 \%)$

Lymphocyte count $\left(\mathbb{1 . 1} 1 \times 10^{9} / \mathrm{L}\right)$

66

$(72.5 \%)$

Lymphocyte ( $\mathbb{2} 20 \%)$

70

$(76.9 \%)$

Haemoglobin

(female囚115 g/L,male $₫ 130 \mathrm{~g} / \mathrm{L}$ )

34

$(37.4 \%)$

Platelet count $\left(\mathbb{1} 125 \times 10^{9} / \mathrm{L}\right)$

18

$(19.8 \%)$

$24(46.2 \%)$

$37(94.9 \%) \quad \bowtie 0.001$

$33(63.5 \%)$

$33(84.6 \%) \quad 0.034$

$31(59.6 \%)$

$39(100 \%)$

$\llbracket 0.001$

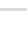

20 (38.5\%)

$14(35.9 \%) \quad 0.748$

$6(11.5 \%)$

$12(30.8 \%) \quad 0.026$

Coagulation function

Prothrombin time ( $(13 \mathrm{~s})$

25

$(27.5 \%)$

$8(15.4 \%)$

$17(43.6 \%)$

0.003

Activated partial thromboplastin time

( $₫ 31.3 \mathrm{~s})$

19

$(20.9 \%)$

$9(17.3 \%)$

$10(25.6 \%)$

0.333

D-dimer $(\mu \mathrm{g} / \mathrm{mL})$

$\bowtie 0.55$

73

$(80.2 \%)$

$\bigotimes 1$

51

$(56.7 \%)$

Biochemical criterion

Creatinine $(₫ 110 \mu \mathrm{mol} / \mathrm{L})$

12

$(13.2 \%)$

$5(9.6 \%)$

$7(17.9 \%)$

0.186

Glomerular filtration rate $(\mathrm{mL} / \mathrm{min})$

$\nabla 90$

34

$(37.4 \%)$
$35(67.3 \%)$

$38(97.4 \%)$

$\varangle 0.001$

$20(38.5 \%)$

$31(79.5 \%)$

$\llbracket 0.001$ 


\begin{tabular}{|c|c|c|c|c|}
\hline & $\begin{array}{l}\text { All patient } \\
(n=91)\end{array}$ & $\begin{array}{l}\text { Survival } \\
\text { group } \\
(n=52)\end{array}$ & $\begin{array}{l}\text { Death } \\
\text { group } \\
(n=39)\end{array}$ & $\begin{array}{l}\mathrm{P} \\
\text { value }\end{array}$ \\
\hline$₫ 60$ & $\begin{array}{l}13 \\
(14.3 \%)\end{array}$ & $4(7.7 \%)$ & $9(23.1 \%)$ & 0.033 \\
\hline Lactate dehydrogenase ( $(250 \mathrm{U} / \mathrm{L})$ & $\begin{array}{l}68 \\
(74.7 \%)\end{array}$ & $34(65.4 \%)$ & $34(87.2 \%)$ & 0.018 \\
\hline \multicolumn{5}{|l|}{ Humoral immunity } \\
\hline Immunoglobulin E ( $\$ 100$ IU/MI) & $\begin{array}{l}14 \\
(15.4 \%)\end{array}$ & $11(21.2 \%)$ & $3(7.7 \%)$ & 0.083 \\
\hline Immunoglobulin M ( $₫ 2.3 \mathrm{~g} / \mathrm{L})$ & $\begin{array}{l}26 \\
(28.6 \%)\end{array}$ & $11(21.2 \%)$ & $15(38.5 \%)$ & 0.025 \\
\hline Immunoglobulin G ( $₫ 16 \mathrm{~g} / \mathrm{L})$ & $\begin{array}{l}67 \\
(73.6 \%)\end{array}$ & $33(64.5 \%)$ & $34(87.2 \%)$ & 0.011 \\
\hline Complement C3 ( $\mathbb{0} 0.9 \mathrm{~g} / \mathrm{L})$ & $\begin{array}{l}28 \\
(30.8 \%)\end{array}$ & $13(25 \%)$ & $15(38.5 \%)$ & 0.069 \\
\hline \multicolumn{5}{|l|}{ Cellular immunity } \\
\hline 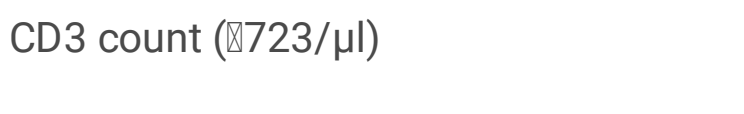 & $\begin{array}{l}66 \\
(72.5 \%)\end{array}$ & $31(59.6 \%)$ & $35(89.7 \%)$ & 0.002 \\
\hline \multicolumn{5}{|l|}{ CD4 count $(/ \mu \mathrm{l})$} \\
\hline$\varangle 404$ & $\begin{array}{l}62 \\
(68.1 \%)\end{array}$ & $27(51.9 \%)$ & $35(89.7 \%)$ & $\varangle 0.001$ \\
\hline$\otimes 200$ & $\begin{array}{l}37 \\
(40.7 \%)\end{array}$ & $12(23.1 \%)$ & $25(64.1 \%)$ & $\varangle 0.001$ \\
\hline CD8 count $(\varangle 220 / \mu \mathrm{l})$ & $\begin{array}{l}59 \\
(31.9 \%)\end{array}$ & $27(51.9 \%)$ & $32(82.1 \%)$ & 0.001 \\
\hline \multicolumn{5}{|l|}{ Myocardial injury index } \\
\hline 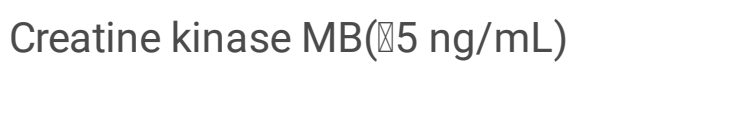 & $\begin{array}{l}27 \\
(29.7 \%)\end{array}$ & $8(15.4 \%)$ & $19(48.7 \%)$ & 0.001 \\
\hline 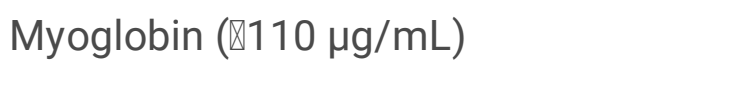 & $\begin{array}{l}30 \\
(33.0 \%)\end{array}$ & $11(21.2 \%)$ & $19(48.7 \%)$ & 0.006 \\
\hline Cardiac troponin $(\otimes 0.04 \mathrm{ng} / \mathrm{mL})$ & $\begin{array}{l}31 \\
(34.1 \%)\end{array}$ & $8(15.4 \%)$ & $23(59.0 \%)$ & $\varangle 0.001$ \\
\hline \multicolumn{5}{|l|}{ Other indexes } \\
\hline B-type natriuretic peptide precursor & $9(9.9 \%)$ & $2(3.8 \%)$ & $7(17.9 \%)$ & 0.07 \\
\hline 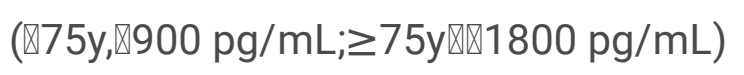 & & & & \\
\hline
\end{tabular}




\begin{tabular}{|c|c|c|c|c|}
\hline & $\begin{array}{l}\text { All patient } \\
(n=91)\end{array}$ & $\begin{array}{l}\text { Survival } \\
\text { group } \\
(n=52)\end{array}$ & $\begin{array}{l}\text { Death } \\
\text { group } \\
(n=39)\end{array}$ & $\begin{array}{l}P \\
\text { value }\end{array}$ \\
\hline High-sensitivity CRP ( $₫ 5 \mathrm{mg} / \mathrm{L})$ & $\begin{array}{l}80 \\
(88.0 \%)\end{array}$ & $42(80.8 \%)$ & $38(97.4 \%)$ & 0.021 \\
\hline Procalcitonin $(0.1 \mathrm{ng} / \mathrm{mL})$ & $\begin{array}{l}17 \\
(18.7 \%)\end{array}$ & $4(7.7 \%)$ & $13(33.3 \%)$ & 0.006 \\
\hline
\end{tabular}

In the death group, almost all patients (97.4\%) have a higher D-dimer level that more than $0.55 \mu \mathrm{g} / \mathrm{mL}$, and $79.7 \%$ patients have a more higher D-dimer level that more than $1 \mu \mathrm{g} / \mathrm{mL}$, and the percentage significantly elevated than in the survival group (pष0.001). The non-survivors also presented a higher proportion of increased prothrombin time $(p=0.003)$, Lactate dehydrogenase $(p=0.022)$. B-type natriuretic peptide precursor $(p=0.07)$, high-sensitivity $\operatorname{CRP}(p=0.021)$, and procalcitonin level $(p=0.006)$ than those survivors. Furthermore, the proportion of abnormal myocardial injury index of death was higher than that of survivors.

Risk factors associated mortality

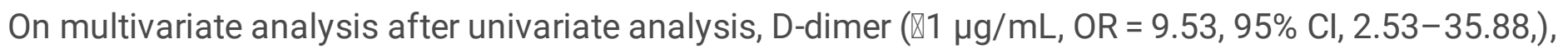

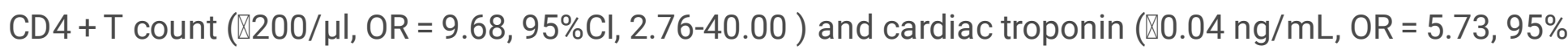
$\mathrm{Cl}, 1.86-17.66)$ were independent risk factors for mortality. (Table 3 ) 
Table 3

Univariate and multivariate analyses of the clinical variables

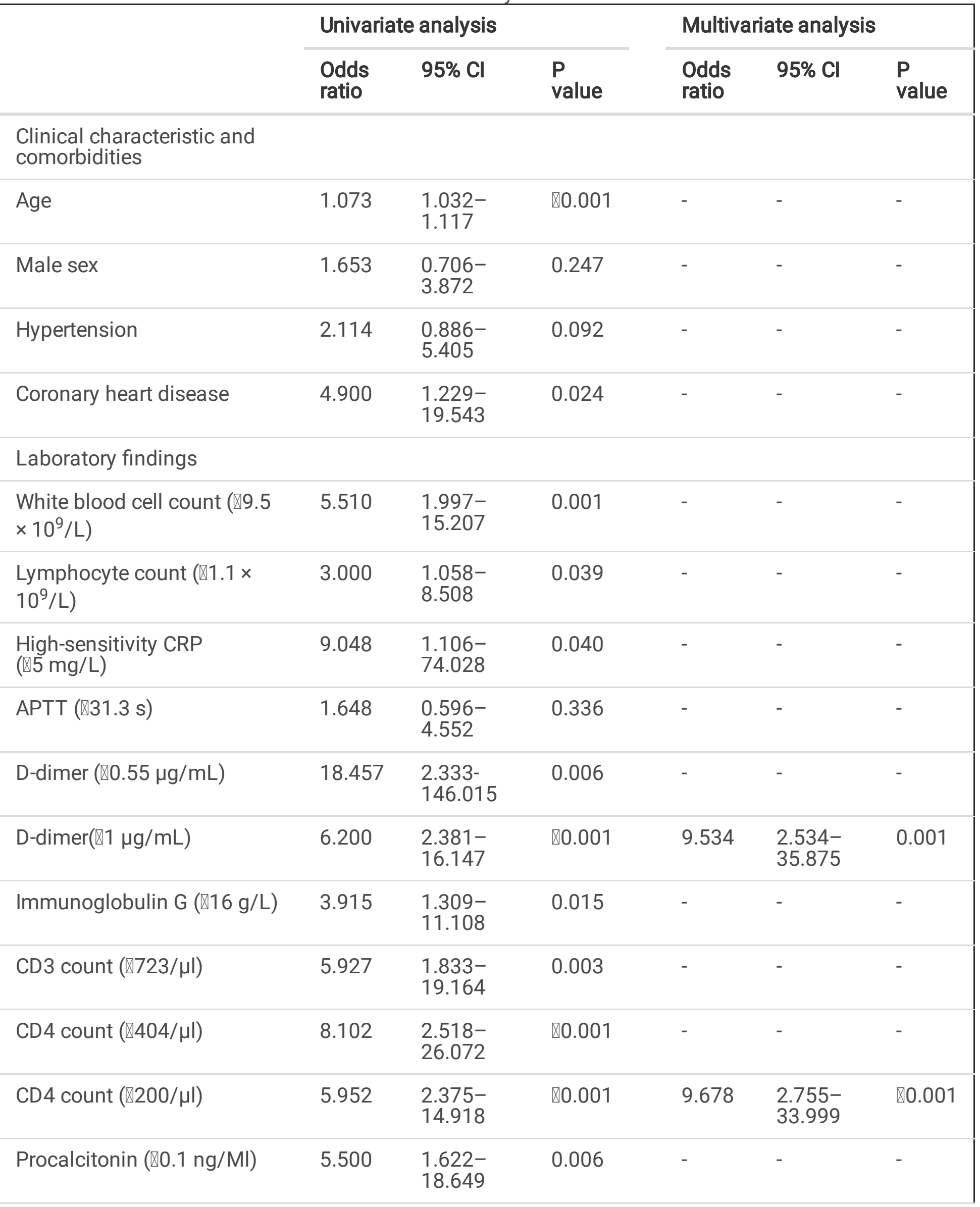




\begin{tabular}{|c|c|c|c|c|c|c|}
\hline & \multicolumn{3}{|c|}{ Univariate analysis } & \multicolumn{3}{|c|}{ Multivariate analysis } \\
\hline & $\begin{array}{l}\text { Odds } \\
\text { ratio }\end{array}$ & $95 \% \mathrm{Cl}$ & $\begin{array}{l}P \\
\text { value }\end{array}$ & $\begin{array}{l}\text { Odds } \\
\text { ratio }\end{array}$ & $95 \% \mathrm{Cl}$ & $\begin{array}{l}P \\
\text { value }\end{array}$ \\
\hline 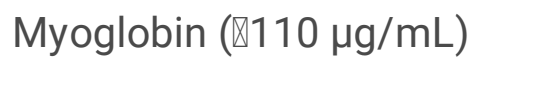 & 3.541 & $\begin{array}{l}1.418- \\
8.841\end{array}$ & 0.007 & - & - & - \\
\hline 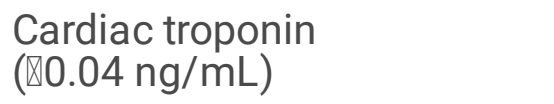 & 7.906 & $\begin{array}{l}2.946- \\
21.218\end{array}$ & $\nabla 0.001$ & 5.725 & $\begin{array}{l}1.857- \\
17.656\end{array}$ & 0.002 \\
\hline 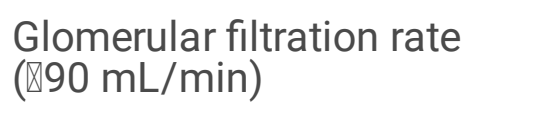 & 3.500 & $\begin{array}{l}1.439- \\
8.515\end{array}$ & 0.006 & - & - & - \\
\hline $\begin{array}{l}\text { Lactate dehydrogenase } \\
(\otimes 250 \mathrm{U} / \mathrm{L})\end{array}$ & 3.600 & $1.2-10.804$ & 0.022 & - & - & - \\
\hline Intravenous immunoglobulin & 3.34 & $\begin{array}{l}0.161- \\
0.932\end{array}$ & 0.034 & & & \\
\hline
\end{tabular}

\section{Discussion}

We report on 91 critically ill inpatients with confirmed SARS-CoV-2 infection. This present retrospective study identified several risk factors for death. In particular, CD4 T cell count less than 200/ $\mu$, d-dimer levels greater than $1 \mu \mathrm{g} / \mathrm{mL}$, and cardiac troponin greater than $0.04 \mathrm{ng} / \mathrm{mL}$ on admission were associated with higher odds of critical in-hospital death. Our study also demonstrated that older age, high-sensitivity C-reactive protein, and lactate dehydrogenase were more commonly seen in severe COVID-19 illness.

This study, to our knowledge, is the first report to investigate the relationship between immune factors and death. Studies have shown that the clinical features of SARS-CoV-2 infection bear some resemblance to SARS-CoV and middle east respiratory syndrome coronavirus (MERS-CoV) infections $[9,10]$. The 2019nCoV infection appears to be initially associated with an increased Th2 response, which might reflect a physiological reaction to curb overt inflammatory responses [11]. Reduced CD4 T cell and CD8 T cell number has been shown in SARS patients [12]. Levels of CD4 T cell and CD8 T cell were lower in severe COVID-19 patients than mild patients [13]. However, the association between CD4 T cell, CD8 T cell and mortality in patients with COVID-19 is limited. In the present study, the results showed that CD 4 T cell and CD8 T cell decreased in critical inpatients with COVID-19. Furthermore, we found that low CD4 T cell level is an independent risk factor for mortality in critical inpatients with COVID-19. Previous study has found that the lower CD4T lymphocyte count is an important predictor for the prognosis and adverse clinical outcomes of cryptococcosis [14]. In HIV/HCV-coinfected individuals the death risk is also related to the level of CD $4+$ cells [15]. The loss of CD 4 Th1 cells directly reduced numbers and function of antiviral CD8 T cell in persistent viral infection [16]. Interestingly, in the present study, we found that there was no significant difference in the treatment of antiviral drug and Methylprednisolone between the two group, while intravenous immunoglobulin was more common in survivors than in death. Whether immunoglobulin treatment can reduce mortality in critical inpatients with COVID-19 should be further to investigated. 
Previous studies have shown that older age, d-dimer greater than $1 \mu \mathrm{g} / \mathrm{mL}$ and greater cardiac troponin are the potential risk factors of inpatients with COVID-19 $[17,18]$. The greater levels of D-dimer and cardiac troponin occurring in non survivors in the current study are similar to the findings of other studies. However, we found that older age is not the independent risk factor for mortality in critical inpatients with COVID-19. The different results between our results and other studies may be related to different criteria for collecting cases. In the present study, all the patients were the critical inpatients with COVID-19 according to the guidance of New coronavirus pneumonia prevention and control program in China (6th edition) [8]. Taken together, these results showed that older age and may be related to the severity of the disease.

Limitations

There are several limitations to this study. First, some patients were transferred from the other hospitals. The different treatment in the early stage of patients may affect the progress of patients. Second, due to the retrospective study design and the limited patients, data from larger populations and multiple centers is warranted to further confirm the outcomes of cellular immunity in COVID-19. Third, in the present study, the surviving patients were not followed up after discharge, so our results only show the risk of mortality during hospitalization.

\section{Conclusions}

The decrease of CD4 T cell is associated with higher risk mortality in critical inpatients with COVID-19. Intravenous immunoglobulin was more common in survivors than in non-survivors.

\section{Abbreviations}

COVID-19

coronavirus disease 2019

SARS-CoV-2

novel severe acute respiratory syndrome coronavirus 2

SOFA

sequential organ failure assessment

COPD

chronic obstructive pulmonary disease

MERS-CoV

middle east respiratory syndrome coronavirus

\section{Declarations}

\section{Ethics approval and consent to participate}


The study was approved by the Ethics Committee of Renmin Hospital of Wuhan University and was conducted according to the principles expressed in the Declaration of Helsinki and its later amendments.

\section{Consent for publication}

Not applicable.

\section{Availability of data and materials}

Data will be made available on request.

\section{Competing interests}

The authors declare that they have no competing interests $\square$

\section{Funding}

This study was funded by the National Natural Science Foundation of China (No.81670303 and No.81970277) and the Science and Technology Key Project on Novel Coronavirus Pneumonia, Hubei Province (project number: 2020FCA002).

\section{Authors' contributions}

JX contributed to statistic, collecting the data. MY contributed to writing the manuscript. WY and XL contributed to data analysis. KH contributed to discussion of the manuscript. QY-Z contributed to design and discussion of the manuscript, DNA sampling. The authors read and approved the final manuscript.

\section{Acknowledgements}

Not applicable.

\section{References}

1. Wu Z, McGoogan JM. Characteristics of and Important Lessons From the Coronavirus Disease 2019 (COVID-19) Outbreak in China: Summary of a Report of 72314 Cases From the Chinese Center for Disease Control and Prevention. JAMA. 2020 Feb 24. doi:10.1001/jama.2020.2648.

2. Wang D, Hu B, Hu C, et al. Clinical Characteristics of 138 Hospitalized Patients With 2019 Novel Coronavirus-Infected Pneumonia in Wuhan, China. JAMA. 2020 Feb 7. doi:10.1001/jama.2020.1585.

3. Yang $X, Y u Y, X u$ J, et al. Clinical course and outcomes of critically ill patients with SARS-CoV-2 pneumonia in Wuhan, China: a single-centered, retrospective, observational study. Lancet Respir Med. 2020 Feb 24. doi:10.1016/S2213-2600(20)30079-5.

4. Chen N, Zhou M, Dong X, et al. Epidemiological and clinical characteristics of 99 cases of 2019 novel coronavirus pneumonia in Wuhan, China: a descriptive study. Lancet. 2020;395(10223):507-13. 
5. Chu H, Zhou J, Wong BH, et al. Middle East Respiratory Syndrome Coronavirus Efficiently Infects Human Primary T Lymphocytes and Activates the Extrinsic and Intrinsic Apoptosis Pathways. J Infect Dis. 2016;213(6):904-14.

6. Liu WJ, Zhao M, Liu K, et al. T-cell immunity of SARS-CoV: Implications for vaccine development against MERS-CoV. Antiviral Res. 2017;137:82-92.

7. Bajema KL, Oster AM, McGovern OL, et al. 2019-nCoV Persons Under Investigation Team; 2019-CoV Persons Under Investigation Team. Persons Evaluated for 2019 Novel Coronavirus - United States, January 2020. MMWR Morb Mortal Wkly Rep. 2020;69:166-70.

8. National Health Commission of China. New coronavirus pneumonia prevention and control program (6th edition). Feb 18, 2020.

9. Assiri A, Al-Tawfiq JA, Al-Rabeeah AA, et al. Epidemiological, demographic, and clinical characteristics of 47 cases of Middle East respiratory syndrome coronavirus disease from Saudi Arabia: a descriptive study. Lancet Infect Dis. 2013;13:752-61.

10. Lee $\mathrm{N}$, Hui $\mathrm{D}$, Wu A, et al. A major outbreak of severe acute respiratory syndrome in Hong Kong. $\mathrm{N}$ Engl J Med. 2003;348:1986-94.

11. Huang C, Wang Y, Li X, et al. Clinical features of patients infected with 2019 novel coronavirus in Wuhan, China. Lancet. 2020;395(10223):497-506.

12. Zhan DR, Tang J. J, et al. The spleen as a target in severe acute respiratory syndrome. FASEB J. 2006;20:2321-8.

13. Wan S, Yi Q, Fan S. J, et al. Characteristics of lymphocyte subsets and cytokines in peripheral blood of 123 hospitalized patients with 2019-nCoV pneumonia (NCP). medRxiv. (2020).

14. Ding Y, Li P, He Q, et al. The CD4 + T-lymphocyte count is an important predictor for the prognosis of cryptococcosis. European Journal of Clinical Microbiology Infectious Diseases. 2017;36:897-904.

15. Hansen S, Kronborg G, Benfield T. Prediction of liver disease, aids, and mortality based on discordant absolute and relative peripheral cd4 $t$ lymphocytes in hiv/hepatitis c virus-coinfected individuals[J]. AIDS Res Hum Retroviruses. 2018;34:1058-66.

16. Snell LM, Osokine I, Yamada DH, De la Fuente JR, Elsaesser HJ, Brooks DG. Overcoming CD4 Th1 cell fate restrictions to sustain antiviral CD8 T cells and control persistent virus infection. Cell reports. 2016;16(12):3286-96.

17. Zhou F, Yu T, Du RH, et al. Clinical Course and Risk Factors for Mortality of Adult Inpatients with COVID-19 in Wuhan, China: A Retrospective Cohort Study. Lancet. 2020. doi:https://doi.org/10.1016/S0140-6736(20)30566-3.

18. Han $\mathrm{H}$, Yang $\mathrm{L}$, Rui L, et al. Prominent changes in blood coagulation of patients with SARS-CoV-2 infection. Clin Chem Lab Med. 2020. doi:10.1515/cclm-2020-0188. 